\title{
PRESERVACION CON PENTACLOROFENOL Y SALES CCA POR BAÑO CALIENTE-FRIO DE LA Tachigalia longiflora Ducke (TANG ARANA)
}

José Sanjurjo Vilchez $\left(^{*}\right)$

\section{RESUMEN}

Con el objeto de examinar las absortiones sólidas de formulaciones al $5 \%$ de concentración de pentaclorofenol y sales CCA, se ensayaron 60 probetas de $5 \times 5 \times 50$ $\mathrm{cm}$ (30 de albura 30 de duramen) de Tachigalia Iongiflora (tangarana), sometiendolas a tratamiento preservador por inmersión, en tres tiempos diferentes, en baño caliente-frío. $L$ a temperatura del Baño Caliente fue de $95+50 \mathrm{C}$ para el Pentaclorofenol y de 45-i-q 50 C para las Sales CCA. La temperatura del Baño Frío para ambos preservantes fue la ambiental, la que durante este ensayo osciló entre $29^{\circ} \mathrm{C}$ y $25^{\circ} \mathrm{C}$.

La absorción sólida estuvo influenciada por el tipo de preservante, con Pentaclorofenol el promedio obtenido fue de $13.21 \mathrm{~kg} / \mathrm{m}^{3}$ y con Sales CCA fue de 4.64 $\mathrm{kg} / \mathrm{m}^{3}$. Igualmente el tipo de madera es un elemento de efectos significativos sobre la absorción sólida pues en el tratamiento con Pentaclorofenol el promedio en la albura fue de $19.68 \mathrm{~kg} / \mathrm{m}^{3}$; mientras que en el duramen fue de $6.73 \mathrm{~kg} / \mathrm{m}^{3}$, en tanto que en la impregnación con CCA fue de $6.67 \mathrm{Kg} / \mathrm{m}^{3}$, en albura y de $2.60 \mathrm{Kg} / \mathrm{m}^{3}$ en duramen. A simismo la acción del tiempo de inmersión en baño caliente es importante, puesto que con el tiempo de inmersión de 6 horas de baño caliente y 9 horas de baño frío (13) se obtuvieron las mayores absorciones, obteniéndose la más alta $\left(21.53 \mathrm{~kg} / \mathrm{m}^{3}\right)$ al tratar muestras de albura con Pentaclorofenol; las menores absorciones se observaron con el tiempo de inmersión de 2 horas de baño caliente y 13 horas de baño frío (11) obteniéndose la más baja $\left(2.12 \mathrm{~kg} / \mathrm{m}^{3}\right)$ al tratar muestras de duramen con Sales CCA .

La absorción fue clasificada como buena en albura y generalmente regular en duramen, la penetración fue catalogada como total en ambos tipos de muestra para el caso del pentaclorofenol mientras que en el tratamiento con sales CCA en el duramen la absorción fue clasificada como mala y la penetración como nula, en tanto que en la albura se observó que la penetración fue regular y la absorción fue, por lo general, también regular.

En base a las absorciones sólidas encontradas y a las penetraciones observadas se clasificó la madera de Tachigolia longiflora como fácil de tratar al impregnarse con pentaclorofenol y difícil de tratar al preservarse con Sales CCA, usando el método de impregnación por baño caliente-frío.

Instituto de Investigaciones de la A mazonia Peruana IIAP, Av. A belardo Quiñones Km. 2.5 A p. 784 Iquitos Perú 
Palabras Caves: preservación, Pentaclorofenol Tachigalia longiflora, Sales CCA, Baño Caliente-F río

\section{ABST RACT}

The solid absorptions of Pentachlorophenol and water-borne CCA formulations at $5 \%$ of concentration using the hot and cold bath preservative treatment in Tachigalia Iongiflora were determined in this work. 60 samples of $5 \times 5 \times 25 \mathrm{~cm}-30$ sapwood and 30 heartwood -were tested to 3 different times of inmersion, being the hot bath temperature $95+50 \mathrm{C}$ for the Pentachlorophenol and $45+50 \mathrm{C}$ for the CCA, and the cold bath temperature the environmental temperature, oscillating from $25 \mathrm{OC}$ to $29 \mathrm{OC}$.

The results showed that the solid absorption was influenced for the type of preservative, in average $13.21 \mathrm{~kg} / \mathrm{m} 3$ of Pentachlomphenol and $4.64 \mathrm{~kg} / \mathrm{m} 3$ of CCA was observed. The kind of wood has a significative effect on the solid absorption, while in sapwood $19.68 \mathrm{~kg} / \mathrm{m} 3$ of Pentachlorophenol and $6.67 \mathrm{~kg} / \mathrm{m} 3$ of CCA was absorbed, in heartwood only $6.73 \mathrm{~kg} / \mathrm{m} 3$ of Pentachlorophenol and $2.60 \mathrm{~kg} / \mathrm{m}^{3}$ of CCA of absorption could be reached. The effect of inmersion time in hot bath is very important, with 6 hour inmersion time in hot bath and 9 hours in cold bath the highest absorption of $21.53 \mathrm{~kg} / \mathrm{m}^{3}$ was obtained in sapwood samples with Pentachlorophenol treatament. The lowest solid absorption of $2.60 \mathrm{Kg} / \mathrm{m} 3$ wasreached $\mathrm{m}$ heartwood samples using CCA in 2-hour hot bath and 13-hour cold bath treatment.

According to the Absorption Classification the impregnation with Pentaclorofenol was classified as good in sapwood and regular in heartwood, with a full penetration in both sapwood and heartwood, while the CCA absorption was regular in sapwood and bad in heartwood, with regular penetration in sapwood and nule in heartwood.

Based on the results the Tachigalia longiflora species can be classified as easyto treat with Pentachlorophenol and difficult to treat with $\mathrm{CCA}$, using the hot and bath impregnation method.

\section{INTRODUCCION}

Para poder utilizar de una mejor manera las propiedades de resistencia y durabilidad de la madera, es conveniente que esté exenta de defectos producidos por insectos, hongos xilófagos y otros factores capaces de afectar sus características fisicomecánicas, echándola a perder y acortando su tiempo de servicio. Una de las formas de asegurarle inmunidad es someterla al tratamiento químico preservador. A nivel local y en un reducido número de aserraderos se realiza el tratamiento preservador utilizando el método de inmersión breve, el que según Hunty Garrat (1962), generalmente proporciona a la madera una protección muy limitada, estas circunstancias permiten entrever la necesidad de difundir procedimientos que, sin ser sofisticados, provean un resguardo más eficaz a la madera, tal es el caso del baño caliente-frío, respecto al cual Krzyewski (1968) manifiesta que es el método más efectivo entre los tratamientos empleando técnicas sin presión. El pentaclorofenol es un preservador oleosoluble muy 
usado, en experimentos de laboratorio con hongos xilófagos e insectos ha demostrado al ta efectividad y bajo costo. Cuando se usa con solventes limpios permite el tratamiento de ventanas, puertas, triplay y otros productos que luego serán pintados (Carlos 1982).

Los componentes de cobre $(\mathrm{Cu})$, cromo $(\mathrm{Cr})$ y arsénico (As), se comportan como uno de los grupos de preservantes más eficientes; en los que actúan el Cu y As como ingredientes tóxicos contra los xilófagos, y al $\mathrm{Cr}$ como agente de fijación; las que no son lixiviables y son apropiadas para maderas destinadas a uso interior o exterior y en contacto con el suelo, permitiendo el empleo de pinturas y barnices. La tangarana (Tachigalia longiflora), es una especie abundante en los bosques de la cuenca amazónica; según inventarios efectuados por Villanueva (1977, 1982, 1984); Villar (1982); Hidalgo (1982); cuya principal limitación es su baja resistencia natural al ataque de hongos e insectos xilófagos, constituyéndose en uno de los factores más restrictivos de su industrialización y posterior comercialización. La preservación de su madera permitirá su incorporación al mercado regional, nacional e internacional. Este estudio fue diseñado para examinar las absorciones sólidas de las formulaciones de pentaclorofenol y sales CCA en la albura y duramen de Tachigalia longifiora (tangarana), sometidas al proceso de baño caliente-frío; asímismo, para determinar el costo promedio de las soluciones de pentaclorofenol y sales CCA.

\section{REVISION DE LITERATURA}

A róstegui (1968), por el método sin presión del baño caliente-frío, encontró una diferencia altamente significativa a favor del pentaclorofenol en comparación con el boliden (derivado CCA), en relación a la penetración y la retención. Observó que la máxima retención $(192.6 \mathrm{~kg} / \mathrm{m} 3)$ para el pentaclorofenol, se presentó en la Simaroruba sp. (Y aguar caspi) y la mínima $(36.8 \mathrm{~kg} / \mathrm{m} 3$ ) en la Pourouma sp. (Quina-quina); para el boliden notó la mayor retención $(57.9 \mathrm{~km} / \mathrm{m} 3$ ) en el Drypetes $\mathrm{sp}$. (Estoraque) y la menor $(7.1 \mathrm{~kg} / \mathrm{m} 3)$ en la Tabebuia sp. (Paliperro); afirma, con un nivel de probabilidad del 95 $\%$, que la penetración media se encuentra para el pentaclorofenol entre $17.8 \%$ y $24.6 \%$ y para el boliden entre $2.5 \%$ y $5.7 \%$ de la sección transversal de las muestras. EI PADT-REFORT/JUNAC (1983) reporta, para el baño caliente-frío realizado con pentaclorofenol disuelto al $5 \%$ en aceite, la más alta absorción en la albura (374 kg/ m3) del Brosimum utile (Sande), proveniente del Ecuador, cuya densidad básica (DB) es 0.40 $\mathrm{gr} / \mathrm{cm} 3$; y las más bajasabsorciones en la albura $(16 \mathrm{~kg} / \mathrm{m} 3)$ del Caryocar coccineum (A Imendro), originario del Perú, que presenta una DB de $0.65 \mathrm{gr} / \mathrm{cm} 3$ y en el duramen (4 $\mathrm{kg} / \mathrm{cm} 3$ ) de Chlorofora tinctoria (M oral fino) procedente del Ecuador, que muestra una $\mathrm{DB}$ de $0.71 \mathrm{gr} / \mathrm{cm}^{3}$. La determinación de la penetración la realizaron en la parte media transversal de las muestras, empleando el reactivo de coloración apropiado para el pentaclorofenol. La evaluación de la madera inmunizada se efectuó con respecto a la absorción cuantitativa y la penetración cualitativa del preservante en la madera, estableciendo luego un índice de permeabilidad relativa en la albura y el duramen de todas las especies examinadas. A rostegui, etal (1979), con el método del baño caliente- 
frío con pentaclorofenol en solución de aceite al 5\% experimentaron en la albura y el duramen de 16 y 19 especies, respectivamente; reportan que la mayor absorción se presenta en la albura $(10.9 \mathrm{~kg} / \mathrm{m} 3)$ y el duramen $(9.6 \mathrm{~kg} / \mathrm{m3})$ de Pseudolmedia laevis (Chimicua), cuya DB es de $0.60 \mathrm{gr} / \mathrm{cm} 3$ y que la menor fue mostrada por la albura $(1.5 \mathrm{~kg} / \mathrm{m} 3)$ de Caryocar glabrum (Almendro), que manifiesta una DB de $0.65 \mathrm{gr} / \mathrm{cm} 3$; y el duramen $(0.2 \mathrm{~kg} / \mathrm{m3})$ de Myroxilon paruiferum. (Estoraque), que muestra una $D B$ de $0.81 \mathrm{gr} / \mathrm{cm} 3$. Referente a la penetración; del análisis de los datos que obtuvieron se deduce que por lo general, a una buena absorción corresponde una penetración total en la albura y Regular en el duramen; así mismo, para una regular o mala absorción es frecuente una penetración irregular tanto en la albura como en el duramen. A róstegui, et al (1985); por inmersión en solución caliente-fría con pentaclorofenol al $5 \%$ de concentración en peso, obtuvieron absorciones entre 16 y307 11/ m3 en la albura; y entre 7 y $297 \mathrm{ll} / \mathrm{m} 3$ en el duramen de las especies en estudio, presentándose los límites inferiores en el Caryocar coccineum (A Imendro) cuya DB es de $0.65 \mathrm{gr} / \mathrm{cm} 3$ y los superiores en la Simarouba amara (M arupa), cuya DB es $0.36 \mathrm{gr} / \mathrm{cm} 3$. La penetración la determinaron en la parte media transversal de las probetas usando los reactivos de coloración recomendados por ITINTEC (1979), reportando penetraciones nulas y totales para las especies mencionadas en el orden respectivo, tanto en albura como en duramen. Espíritu (1985), ensayó muestras de Lacythis peruviana M achimango blanco mediante el tratamiento por baño caliente-frío con pentaclorofenol disuelto en petróleo diesel $\mathrm{N} N 2$, a diversas concentraciones. Informa que obtuvo los mejores resultados con una concentración del $6 \%$, ya que a este nivel logró la mayor retención (100.28 $\mathrm{kg} / \mathrm{m} 3)$ y la más alta penetración radial $(95.8 \%)$, con respecto al espesor de la muestra. Según A róstegui (1975), la densidad básica (DB) de esta especie es $0.72 \mathrm{~g} / \mathrm{cm} 3$.

\section{MATERIALES Y METODOS}

\subsection{Lugar de Ejecución}

El material de ensayo fue obtenido en el bosque experimental del CENTRO DE INVESTIGACION Y ENSEÑANZA FORESTAL Pto. Almendras (CIEFOR - Pto. A Imendras), cerca a Iquitos, LoretoPerú, en donde se ubicaron arbitrariamente 5 árboles que exhibieron condiciones fitosanitarias óptimas y fuste recto, con un DAP promedio de $55.0 \mathrm{~cm}$ y alturas totales entre 17 y $25 \mathrm{~m}$.

\subsection{Materiales y Equipo}

Los tanques de impregnación se diseñaron y se construyeron de un tambor de combustible, de aproximadamente 200 It. de capacidad, el que fue cortado longitudinalmente por la mitad y las partes obtenidas acondicionadas adecuadamente. El tanque para el baño caliente estuvo accionado por electricidad cuyo flujo fue controlado por un termostato. La Figura 1 muestra el esquema completo del dispositivo. La Figura 2 presenta el esquema del circuito eléctrico.

Para el tratamiento de preservación se utilizaron los preservantes siguientes: 


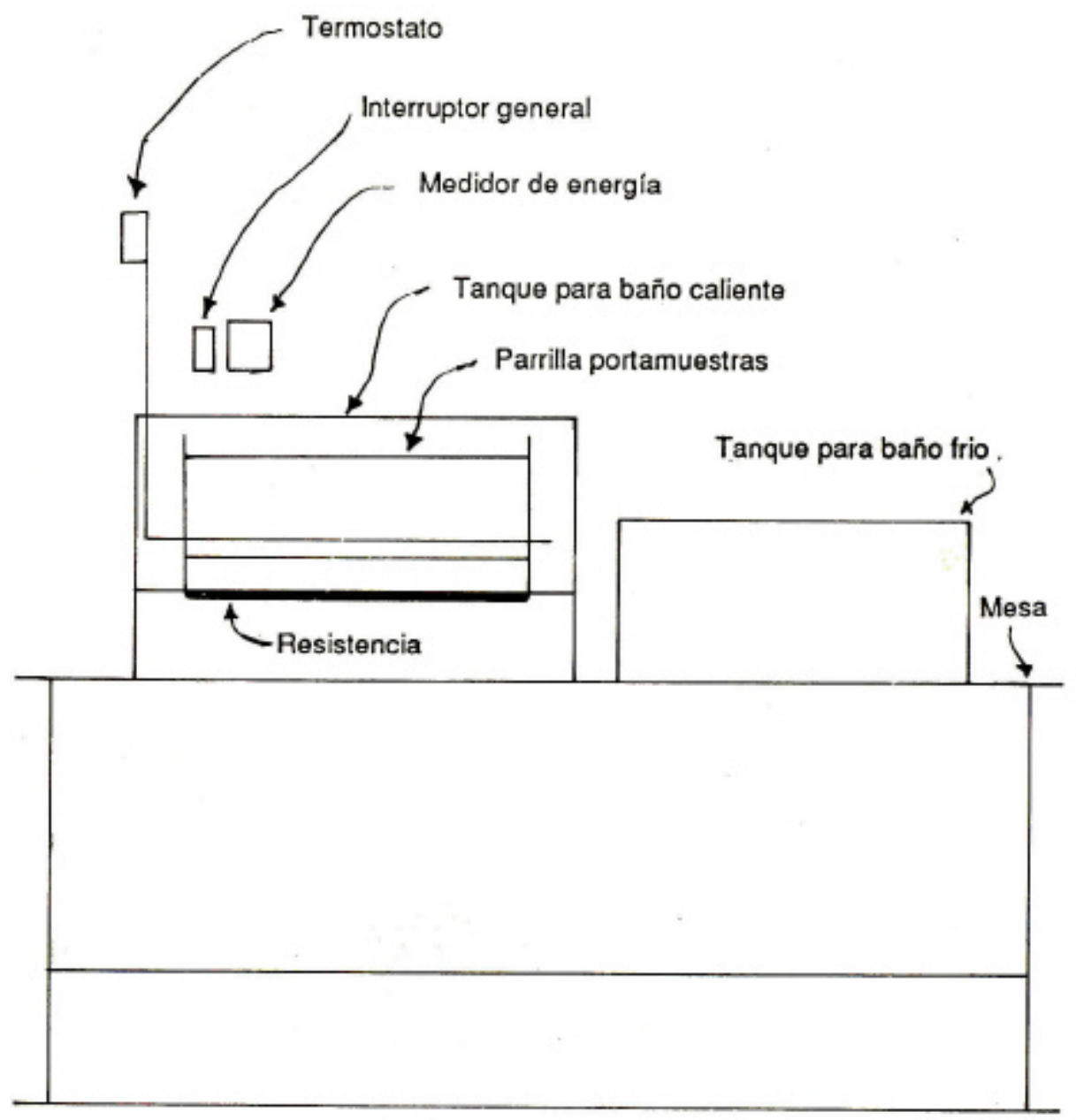

FIGURAS № 1: Situación esquemática del equipo de impregnación 


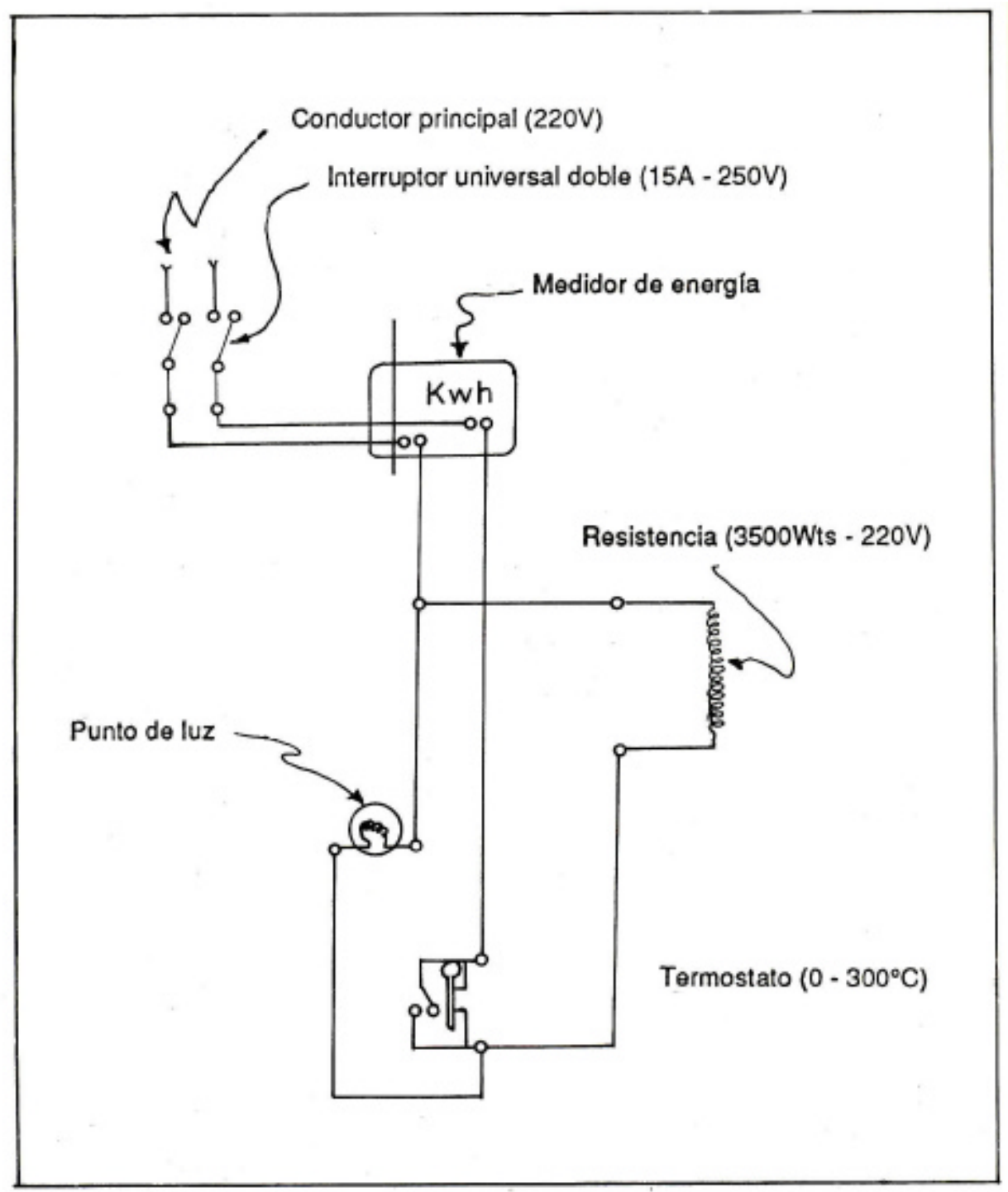

FIGURAS $\mathrm{N}^{2}$ 2: Esquema del circuito eléctrico 
Solución pentaclorofenólica (DURAMAD / JE-10) al $55 \%$ a la que se agregó petróleo diesel N2 2 hasta obtener una solución de ensayo al $5 \%$ de concentración

Sal CCA tipo B en pasta disuelta en una cantidad de agua corriente suficiente para conseguir la concentración de ensayo del $5 \%$.

\subsection{Métodos}

U bicados los árboles se procedió a tumbarlos y a trocearlos, a partir de la linea de corte, cada $80 \mathrm{~cm}$ en toda su longitud comercial; luego, se prepararon cuartones que tuvieron albura y/o duramen, y cuya sección transversal fue aproximadamente de $20 \times 20 \mathrm{~cm}$ y su eje mayor igual y paralelo al eje longitudinal de las trozas, seleccionándose 95 de ellos. Estos fueron aserrados en una sierra ligera de cinta, obteniéndose probetas de $7 \times 7 \mathrm{~cm}$ y de largo igual al de las trozas iniciales; en este proceso, no se hicieron mayores esfuerzos por controlar la orientación de los anillos de crecimiento debido a que en la madera de dimensiones normales se presentan en forma arbitraria. Estas piezas fueron sometidas a secado al aire libre bajo cobertizo hasta obtener un contenido de humedad en estado de equilibrio con el del medio ambiente. A lcanzada esta condición, fueron reaserradas hasta conseguir muestras de $5 \times 5$ x $50 \mathrm{~cm}$; ; luego, se eligieron 60 muestras de ensayo (30 de albura y 30 de duramen). En esta etapa, se excluyeron todas aquellas probetas que presentaron desarrollos reductores de la permeabilidad, tal como los nudos, o defectos que propiciaran una mayor impregnación, tal como rajaduras y perforaciones; se asumió que cualquier pequeño defecto pasado por alto es independiente. Finalmente, se asignaron 5 muestras a cada tratamiento experimental.

El tratamiento de preservación por inmersión en baño caliente frío, se realizó considerando un tiempo total de 15 horas, con los niveles siguientes:

$\begin{array}{lcl}\text { NIVEL } & \text { BAÑO CALIENTE } & \text { BAÑO FRIO } \\ \text { TI } & 2 \text { horas } & 13 \text { horas } \\ 12 & 4 \text { horas } & 11 \text { horas } \\ 13 & 6 \text { horas } & 9 \text { horas }\end{array}$

L a temperatura empleada para el baño caliente con pcntaclorofenol fue de $95+$ $50 \mathrm{C}$. M ientras que con sales CCA fue de $45+50 \mathrm{C}$. La inmersión en baño frío, con ambos preservantes, se realizó a la temperatura ambiental.

\subsection{Procedimiento}

A proximadamente 15 días antes del ensayo de impregnación, se sellaron los extremos de las probetas, con pintura de aluminio, para la sal 
CCA; y para el pentaclorofenol, con un formador de empaquetaduras el que luego de ser aplicado fue presionado con una prensa de carpintería o «sargenta», con la finalidad de disminuir la impregnación logitudinal en las muestras; luego, inmediatamente antes de iniciar el tratamiento, se pesaron.

Para cada tiempo de inmersión total y para cada preservante en particular, se preparó la solución a la concentración de ensayo en cantidad suficiente para ambos tanques.

Utilizando energía eléctrica y bajo el control del termostato se calentó la solución de uno de los tanques con el objeto de elevar la temperatura hasta el punto establecido para efectuar el baño caliente. L uego de haberse sumergido las muestras en esta solución, con el termómetro y en lapsos de tiempo arbitrarios, se fue verificando la temperatura de la disolución.

Al concluir el tiempo de inmersión determinado para el baño caliente, se extrajeron las muestras ( 5 de albura y 5 de duramen por cada tiempo total) e inmediatamente se sumergieron en el tanque para el baño frío, donde se dejaron estar durante el tiempo de inmersión establecido; al concluir éste, se extrajeron para dejarlos escurrir y secar superficialmente durante 2 horas más 30 minutos, al final del cual se pesaron. Finalmente, fueron sometidos a un proceso de secado al aire libre bajo cobertizo, con la finalidad de lograr la fijación del CCA y pentaclorofenol, para lo cual arbitrariamente se consideró un tiempo mínimo de 60 días.

\subsection{Evaluación}

Se estimaron valores de absorción y penetración; para ambos preservantes, se calculó la absorción en base a las consideraciones siguientes:

Absorción líquida: Que se refiere a la absorción de disolución (soluto + solvente), según Torres (1968) está definida por la expresión:

$A e=\frac{P 2-P I}{V} \times 1000 ;$ expresado en $/ \mathrm{m}^{3}+c / m^{3}$

Esta absorción fue calculada con fines informativos.

Absorción de producto sólido: Se refiere a la absorción de sólidos de pentaclorofenol o de sales CCA. 
Para el pentaclorofenol se usó la expresión recomendada por Torres (1968), que es similar a la propuesta por ITINTEC (1979) para las sales CCA:

$$
A s=\frac{C *(P 2-P I) X}{V} 10 ; \text { expresado en } \mathrm{Kg} / \mathrm{m}
$$

Donde :

$\begin{array}{lll}\text { As } & : & \text { Absorción sólida }(\mathrm{Kg} / \mathrm{m} 3) \\ \text { C } & : & \text { Concentración del preservante }(\%) \\ \mathrm{Pl}, \mathrm{P} 2 & : & \text { Pesos }(\mathrm{g}) \text { inicial y final, respectivamente } \\ \mathrm{V} & : & \text { Volumen }(\mathrm{cm} 3) \text { de la probeta }\end{array}$

El desconocimiento de un método para cuantificar la superficie impregnada por el preservante, en la sección media transversal de la probeta, permitió proponer el procedimiento en el cual se asumiría que la distribución del preservante observado con los reactivos de coloración en dicha sección, es uniforme, con la limitación deque esta penetración fuera susceptible de medirse y adaptarse a una figura geométrica cualquiera. La magnitud de la superficie impregnada se calcularía como un porcentaje del área de la sección media transversal, con la expresión basada en la regla de tres simple, que se define como:

$\%=\frac{A i}{A} \times 100$ donde;

$\%$ P : Penetración porcentual

$\mathrm{Ai}:$ : rea (cm2) impregnada, se calcular con la relación

$\mathrm{Ai} \quad=\mathrm{A}-\mathrm{M} \mathrm{x}$ donde;

A : A rea $\left(\mathrm{cm}^{2}\right)$ de la sección media transversal

Mx : A rea (cm2) no impregnada de la sección media transversal, se derivaría de una serie de 6 medidas de las zonas impregnadas, cuyo promedio sería tomado como el lado de un cuadrado, si este fuera el caso.

La clasificación de la madera, se realizó en función de la absorción sólida y de la distribución (penetración) del preservante observada en la sección media transversal de las probetas, de acuerdo a las recomendaciones de ITINTEC (1979). 


\section{RESULTADOS}

La Tabla 1, muestra las absorciones sólidas en cada una de las repeticiones, para cada combinación tiempo de inmersión-preservante-origen de la muestra.

Se observaron bajos coeficientes de variación en los volúmenes de las probetas, mientras que en las diferencias de pesos éstos fueron considerables; así mismo, se notó que los promedios de las absorciones sólidas de las muestras, en general, se incrementaron al aumentar el tiempo de inmersión en baño caliente, siendo menores en las muestras de duramen y considerablemente más bajas en el tratamiento con sales CCA, apreciándose valores altos en los coeficientes de variación. En la impregnación con pentaclorofenol, el área impregnada (Ai) promedio fue igual al área de la sección media transversal (A); sin embargo, en el caso del CCA no alcanzó valores de consideración.

La clasificación de las absorciones sólidas y de la distribución (penetración) del preservante en la sección media transversal de las probetas, para cada tiempo de inmersión, y específicamente para cada nivel de origen de la muestra, permitió observar una mayor frecuencia de absorciones buenas (AB), así como la predominancia de penetraciones totales (PT) en la impregnación con pentaclorofenol, en tanto que en el tratamiento con sales CCA, el mayor número de absorciones son malas (AM) y las penetraciones entre nulas (PN) y regulares (PR).

EI ANVA de la Tabla 2 muestra la variabilidad, con un nivel de confianza del $5 \%$, de la absorción sólida, debido a los efectos principales de los factores tiempo de inmersión, preservante, origen de la Muestra, así como los referentes a las interacciones respectivas.

El cálculo de los costos, particularmente para cada solución preservante, se realizó en función de consideraciones diferentes. 


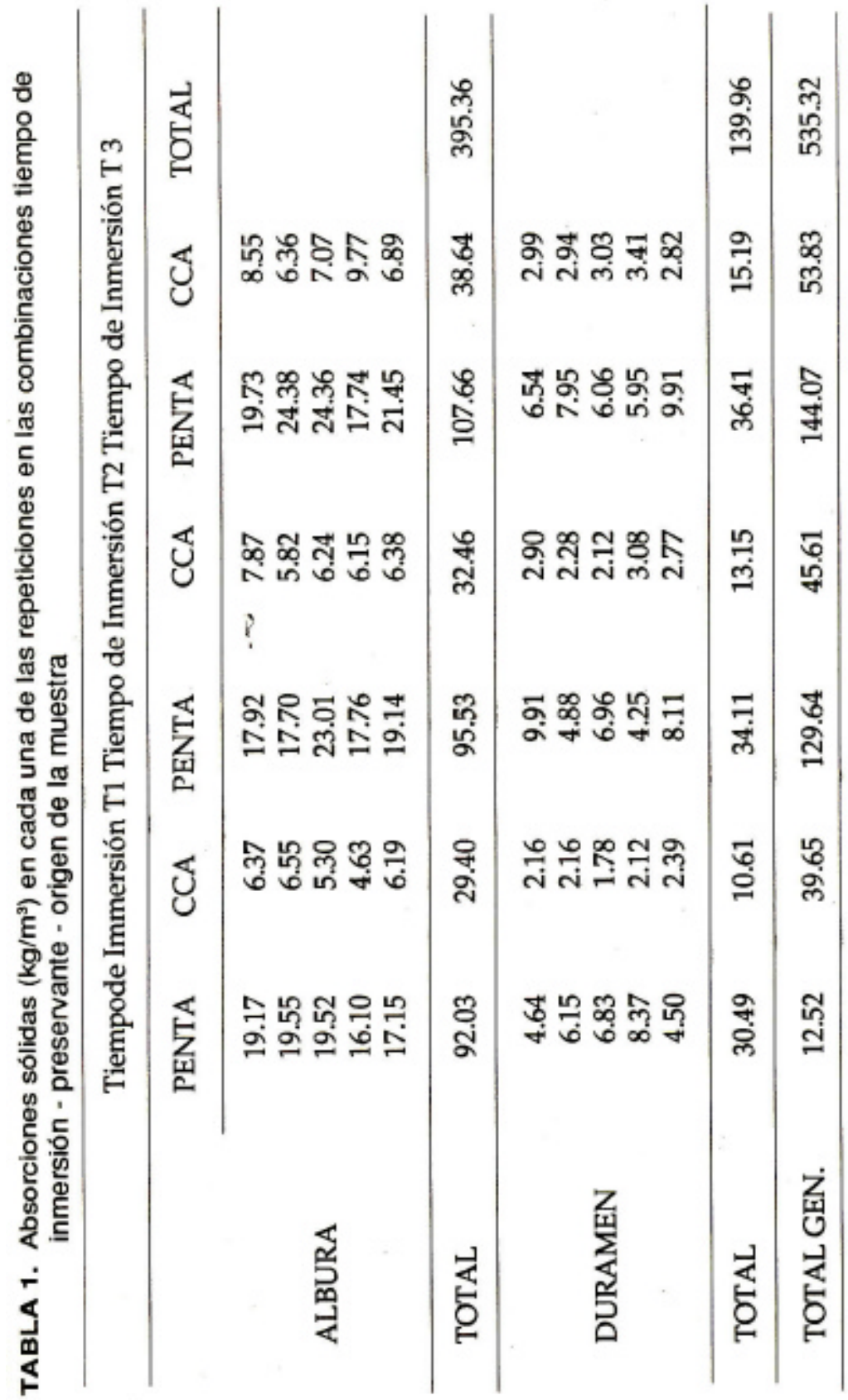




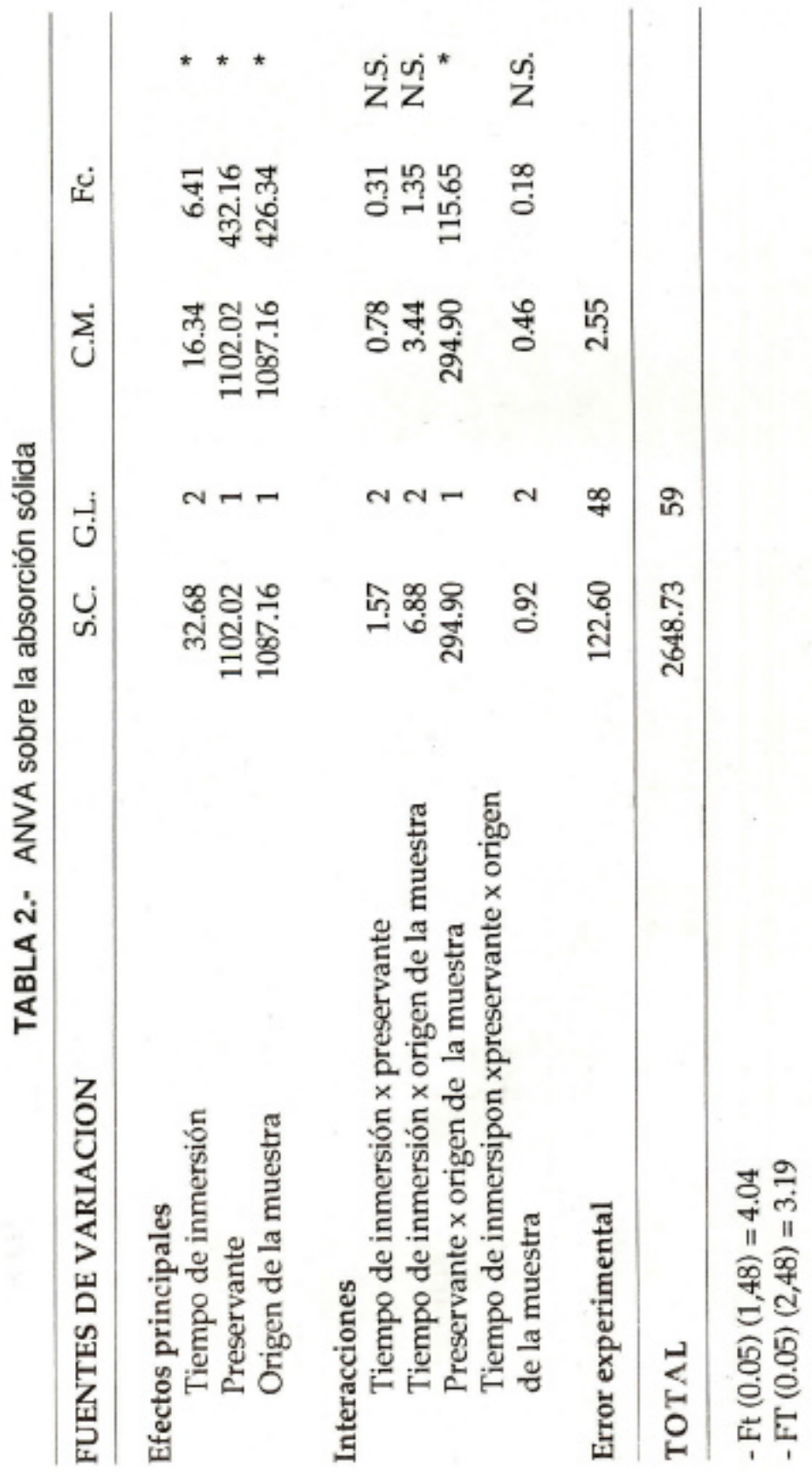




\section{DISCUSION}

El método empleado para impregnar la madera de Tachigalia longiflora se considera adecuado a las características de la misma, por cuanto se obtuvieron resultados que reflejan la permeabilidad de las muestras tratadas, en especial de la madera de albura. Los bajos valores de los coeficientes de variación de los volúmenes de las probetas, permiten afirmar que las absorciones sólidas exhibidas por las probetas de ensayo, no fueron influenciadas por la diferencia de dimensiones entre las mismas.

L os resultados obtenidos dejan entrever que la absorción sólida depende del tipo de preservante, pues mientras el promedio con pentaclorofenol (PENTA) fue de 13.21 $\mathrm{kg} / \mathrm{m}^{3}$ con el CCA fue de $4.64 \mathrm{~kg} / \mathrm{m3}$; los que estadísticamente son significativamente diferentes. Estas respuestas desiguales se deben fundamentalmente a la naturaleza de cada preservante y de los solventes respectivos; pues cada uno de ellos presenta particularidades principalmente en la viscosidad y densidad, así como en las propiedades de las soluciones resultantes; que determinaron los límites sobre los que se aplicaron otros agentes que influyeron marcadamente en los resultados; tal es el caso de la temperatura del baño caliente que; en el tratamiento con pentaclorofenol, fue de $95+5$ OC yen el tratamiento con CCA de $45+5 \stackrel{\circ}{ }$, para evitar la precipitación de los compuestos activos. Estos resultados concuerdan con los lineamientos teóricos de Hunt y Garrat (1972), quienes señalan que la acción de estos factores es significativa.

I gualmente, el tipo de madera es un elemento que afectó absorción sólida de ambos preservantes. En el tratamiento con pentaclorofenol, el promedio en la albura fue de $19.68 \mathrm{~kg} / \mathrm{m} 3$, mientras que la producida en el duramen fue de $6.73 \mathrm{~kg} / \mathrm{m} 3$; en tanto que en la impregnación con CC A fue de $6.67 \mathrm{Kg} / \mathrm{m3}$ en albura y de $2.60 \mathrm{Kg} / \mathrm{m3}$ en duramen, valores entre los que hay una diferencia aproximada de tres veces; y entre los que existe una diferencia estadística significativa, coincidiendo con las observaciones de Tuset y Durn (1979), se puede afirmar que estos resultados disparejos se deben a la diversidad existente entre las formas celulares de la albura y el duramen de la Tachigalia longiflora, las que se manifiestan básicamente en los poros/vasos, cuyas aberturas tangenciales permiten una mejor circulación de líquidos en la albura, mientras que en el duramen está restringida por la considerable reducción de las mismas debido a la lignificación de las paredes y a la presencia de tilosis en los vasos. A simismo, la acción sobre la absorción sólida del tiempo de inmersión en baño caliente, es importante. La Figura 3 muestra que un cambio de nivel en el tiempo de inmersión ocasiona un efecto positivo sobre la absorción sólida, la que aumenta al cambiar este tiempo de 2 a 4 y de 4 a 6 horas. EI ANVA (Tabla 3), de los efectos simples del tiempo de inmersión sobre la absorción sólida muestra diferencias significativas en el tratamiento de muestras de albura con pentaclorofenol. La prueba de Duncan (Tabla 4) corrobora que el mayor efecto se da con el tiempo de inmersión de 6 horas de baño caliente y 9 horas de baño frío (13). Las absorciones sólidas obtenidas en este nivel son las mayores, resultando la más al ta $(21.53 \mathrm{~kg} / \mathrm{m} 3)$ al tratar muestras de albura con Pcntaclorofenol. Confirmando la opinión de Hunt y Garrat (1972) se puede afirmar que esta mayor absorción sólida es 
respuesta a la mayor duración (6horas) de la inmersión en baño caliente. Aún cuando curvas lineales o no lineales pueden ser adecuadas a los datos de absorción sólida versus el nivel del tiempo de inmersión en baño caliente, el experimento no fue diseñado para proveer estimados óptimos de tales curvas; sin embargo se utilizaron resultados de curvas de respuesta para los componentes lineal y cuadrático, a fin de examinar la tendencia de la absorción sólida de pentaclorofenol en madera de albura. Los valores encontrados revelaron tendencias lineales y curvilíneas positivas, resultando la primera significativa al nivel del $5 \%$ de probabilidad, por lo que se procedió a determinar la respectiva ecuación de regresión: $Y=16.56333+X(0.78)$, la misma que debe ser observada con cautela por cuanto en los cálculos no se consideró el efecto derivado de la saturación de las fibras. En la impregnación con pentaclorofenol, las absorciones sólidas medias (A bs) fueron clasificadas predominantemente como buenas ( $B$ ), por cuanto se encuentran en un rango que varía entre 7.28 y $21.53 \mathrm{~kg} / \mathrm{m} 3$, superando el mínimo de 7 $\mathrm{kg} / \mathrm{m} 3$ recomendado por ITINTEC (1979) para la categoría antes señalada; las clasificadas como absorciones regulares (AR), incurren en ésta, pues los valores medios considerados (6.10 y $6.82 \mathrm{~kg} / \mathrm{m} 3$ ) cumplen la condición indicada para la clase antes mencionada, mientras que en el tratamiento con sales CCA, las absorciones sólidas medias fueron clasificadas generalmente como absorciones malas (AM), por cuanto varían entre 2.12 y $5.81 \mathrm{~kg} / \mathrm{m} 3$ por debajo del valor de $6 \mathrm{~kg} / \mathrm{m} 3$ mínimo en dicho rango, y como absorciones regulares (AR) los valores medios $(6.49$ y $7.73 \mathrm{~kg} / \mathrm{m} 3$ ) que están en el intervalo que define a esta categoría. La metodología propuesta para cuantificar porcentualmente el área de la sección media transversal penetrada por el preservante, se aplicó únicamente al tratamiento con pentaclorofenol, debido a que la distribución del preservante en dicha sección de las muestras de albura y duramen permitió establecer una relación de simetría con el área del cuadrado; asumiéndose que la penetración del CCA no ofrece aptitud para ser medida, ya que su distribución no se adaptó a ningún model o distintivo que permita su asociación a áreas geométricas. El cálculo del costo de cada una de las soluciones preservantes, dejó entrever una marcada diferencia entre los mismos, pues mientras que en el tratamiento con sales CCA el costo es de 1.41 Dólares A mericanos por pie tablar, en la impregnación con pentaclorofenol se incrementa en aproximadamente $200 \%$. Los costos antes mencionados fueron reducidos drásticamente al ser calculados bajo otras consideraciones, así en el tratamiento con pentaclorofenol el decremento es cerca de $79 \%$, en tanto que en la impregnación con sales CCA es alrededor de 77\%; asimismo, el incremento de costos entre ambas soluciones preservantes disminuye a $175 \%$. Las consideraciones tomadas en cuenta se refieren al uso de solución preservante únicamente para el baño frío, pues durante el baño caliente no se producen absorciones y penetraciones significativas (Tuset y Durand (1979), pudiendo emplearse en esta fase aceite en desuso para el tratamiento con pentaclorofenol y solamente agua en la impregnación con sales CCA; igualmente, están referidos al uso de un recipiente de dimensiones menores al utilizado originalmente para el baño frío, por cuanto se advirtió que el tanque empleado provocó el uso en exceso de aproximadamente 8.51 de solución preservante 
TABLA 3.- ANVA de los efectos simples sobre la absorción sólida

FUENTES DE VARIACION S.C. $\quad$ G.L. C.M. Fc.

Efectos simples del tiempo de Inmersión

$\begin{array}{lllrll}\text { En el PENTA } & 24.11 & 2 & 12.06 & 4.72 & * \\ \text { En el CCA } & 10.14 & 2 & 5.07 & 1.99 & \text { N.S. } \\ \text { En la ALBURA } & 33.99 & 2 & 17.00 & 6.67 & * \\ \text { En el DURAMEN } & 5.57 & 2 & 2.78 & 1.09 & \text { N.S. }\end{array}$

$\begin{array}{llll}\text { Error experimental } & 122.60 & 48 & 2.55\end{array}$

$\begin{array}{lll} & 196.41 & 56\end{array}$

-Ft. $(0.05)(1,48)=4.04$

- Ft $(0.05)(2,48)=3.19$

TABLA 4.- Resultado artenidos en la Prueba DUNCAN

PRESERVANTE

Pentaclorofenol > Sales CCA

TIEMPOS DE INMERSION

Pentaclorofenol

$\begin{array}{lllll}\text { Albura } & \mathrm{T} 3>\mathrm{T} 2=\mathrm{T} 1 \\ \text { Duramen } & \mathrm{T} 3=\mathrm{T} 2=\mathrm{T} 1\end{array}$

Sales CCA

$\begin{array}{llllll}\text { Albura } & \mathrm{T} 3= & \mathrm{T} 2= & \mathrm{T} 1 \\ \text { Duramen } & \mathrm{T} 3=\mathrm{T} 2= & \mathrm{T} 1\end{array}$

ORIGEN DE LA MUESTRA

Pentaclorofenol

Albura $>$ Duramen

Sales CCA

Albura $>$ Duramen

Los símbolos < y > denotan la primacía de un tratamiento, esto es menor que o menor que otro al nivel de confianza del $5 \%$, un signo igual indica inexistencia de significancia al nivel aludido. 


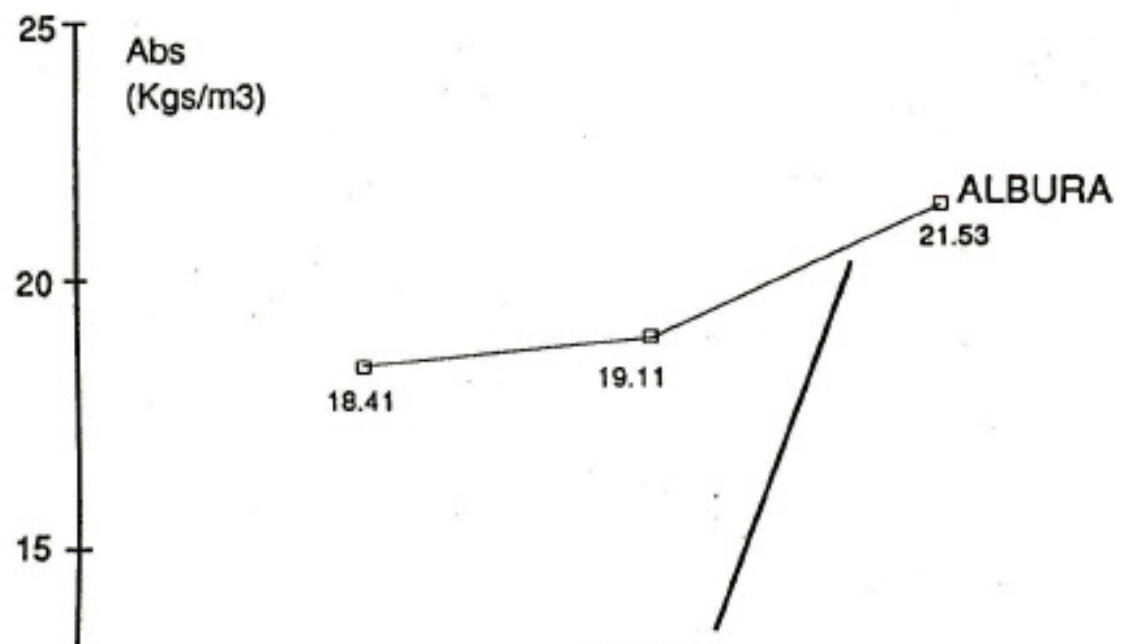

\section{PENTA}

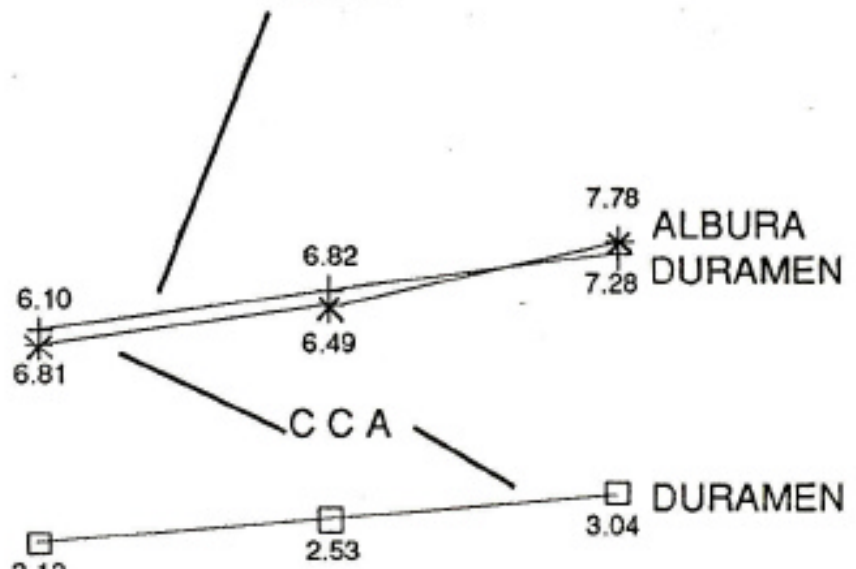

2.12

0
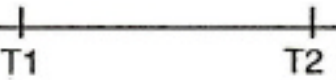

T3

Fig. 3: Efectos debidos a la albura y al duramen sobre la absorción sólida para los preservantes penta y CCA, expresado como promedio de las observaciones en la combinación Tiempo de Inmersión - Tipo de Preservante - Origen de la muestra. 


\section{CONCLUSIONES}

L as absorciones sólidas encontradas y las penetraciones observadas permiten clasificar la madera de Tachigalia longiflora en función de su permeabilidad, como madera fácil de tratar (FT) en la impregnación con pentaclorofenol y difícil de tratar (DI) en el procedimiento con sales CCA.

La absorción sólida media de pentaclorofenol $(13.21 \mathrm{~kg} / \mathrm{ni})$ fue significativamente superior a la lograda con sales CCA (4.64 kg/ni3).

En el tiempo de inmersión de 6horas de baño caliente y 9 horas de baño frío (13) se obtuvierón absorciones sólidas que en promedio fueron pentaclorofenol 14.41 $\mathrm{kg} / \mathrm{m} 3$, sales CCA $5.38 \mathrm{~kg} / \mathrm{m} 3$, valores mayores a los logrados en los dos tiempos restantes ( $12=4$ horas de baño caliente y $\|$ horas de baño frío; $\mathrm{T} 1=2$ horas de baño caliente y13 horas de baño frío); resultando estadísticamente superior únicamente en el tratamiento de muestras de albura con pentaclorofenol.

L as absorciones sólidas medias alcanzadas por las muestras de albura fueron mayores que las de duramen, los promedios fueron pentaclorofenol $19.68 \mathrm{~kg} / \mathrm{m} 3 \mathrm{y} 6.73$ $\mathrm{kg} / \mathrm{ni} 3$, sales CCA $6.67 \mathrm{~kg} / \mathrm{ni} 3$ y $2.60 \mathrm{~kg} / \mathrm{ni} 3$ respectivamente, las que fueron significativamente diferentes. Las absorciones sólidas son respuestas, al tiempo de inmersión en baño caliente, de tendencia lineal positiva; esta tendencia y los parametros del modelo estimado, son apropiados solamente bajo las características asumidas en este ensayo y necesitan reajustes en condiciones diferentes.

Finalmente, el ensayo mostró que el costo de la solución pentaclorofenólica US\$ 4.12 por pie tablar) fue mayor que el costo de la solución de CCA (US\$ 1.41 por pie tablar).

\section{RECOMENDACIONES}

Al preservar la madera con productos venenosos tales como el pentaclorofenol y las sales CCA, se recomienda proceder de acuerdo a un plan cautelosamente preparado, que contemple fundamentalmente la eliminación de las soluciones residuales; puesto que al depender éstos de su toxicidad su descarte inadecuado podría tener efectos adversos sobre el medio ambiente. Para preservar la madera de Tachiagalia longiflora, se recomienda el método de baño caliente - frío con pentaclorofenol, sometiendo a la madera a 6 horas de baño caliente y 9 horas de baño frío.

Se recomienda efectuar estudios para determinar si las sales CCA empleadas en la preservación por baño caliente - frío a las temperaturas consideradas en este ensayo, proveen a la madera protección apropiada.

Siendo el costo de la solución preservante uno de los factores que determinan la acepción del método de preservado, se recomienda dar la importancia necesaria a las consideraciones que permitirán su reducción. En lo referente al baño caliente - frío debe evítarse el uso de soluciones que signifiquen costos durante el baño caliente; asimismo, deber preveerse la utilización adecuada de la capacidad de los tanques de impregnación. 
Es recomendable realizar estudios de factibilidad de una planta piloto a escala industrial para determinar si la producción de madera preservada por inmersión en baño caliente frío es una inversión económicamente rentable.

Finalmente, se recomienda efectuar ensayos para determinar silos preservantes utilizados en este estudio y bajo las condiciones del mismo proveen protección adecuada o sufren alguna pérdida de eficacia a través del tiempo, asi como realizar experimentos de tolerancia a la exposición a los factores atmosféricos; llevar a cabo pruebas para señalar los efectos sobre la pintabilidad y las características físicas y mecánicas de Tachigalia longiflora Ducke.

\section{BIBLIOGRAFIA}

AROSTEGUI, A. 1968. Penetración y Retención de Penta-clorofenol y Boliden mediante Tratamiento sin Presión en Maderas de Tingo María - (UTCF). Revista Forestal del Perú. 2(1)22-23.

AROSTEGUI,.A. (Coordinador). 1975. Estudio Tecnológico de M aderas del Perú. V ol. III. Características Tecnológicas y U sos de la M adera de 40 Especies del Bosque $\mathrm{N}$ acional A lexander $\mathrm{V}$ on Humboldt. Lima. U niversidad $\mathrm{N}$ acional A graria - M inisterio de A gricultura. 171 pp.

AROSTEGUI, A. (Coordinador). 1979. Estudio Integral de la Madera Para la Construcción. Lima. Universidad Nacional A graria - M inisterio de A gricultura. 2a.Ed. 166pp.

CARLOS, L. 1982. Pentaclorofenol. Madera. Boletin Técnico. Informativo sobre M aderas. Colombia. 1(2): 19-23.

ESPIRITU, J. 1985. Penetración y Retención del Pentaclorofenol a Diferentes Concentraciones mediante Tratámiento sin Presión en Lecythis peruviana (Machimango blanco). Tesis Ing. For. Iquitos. Perú. UNAP. Facultad de Ingeniería Forestal. 55 pp.

HIDALGO, W. 1982. Evaluación Estructural de un Bosque Húmedo Tropical en Requena - Perú. Tesis Ing. For. Iquitos. Perú. UNAP. Facultad de Ingeniería Forestal. $142 \mathrm{pp}$.

HUNT, M. yA. GARRAT 1\%2. Preservación de la Madera. Trad. de la la. Ed. en Inglés por A. Sanchis. M adrid. Salvat. 186 pp. 
INSTITUTO DE INVESTIGACION TECNOLOGICA INDUSTRIAL Y DE NORMAS TECNICAS. 1979. Clasificación de M aderas N acionales por sus Características de Preservación. Norma ITINTEC 251.032. Lima. 4 pp.

KRZYZEWSKI,J. 1968. Field Evaluation of Fence Posts Creosoted by Thermal Process. Ottawa. Canada. Eastern Forest Products Laboratory. Report OPX 25E. $12 \mathrm{pp}$.

PADT-REFORT/JUNAC. 1983. Secado y Preservación de Maderas del Grupo A ndino. J unta del A cuerdo de Cartagena. Lima. 151 pp.

TORRES,J. 1968. Conservación de M aderas en su A specto Práctico. M adrid. Instituto Forestal de Investigación y Experiencias. p. irr.

TUSET, R. y F.DURAN 1979. M anual de Maderas Comerciales, Equipos y Procesos de Utilización (A serrado, Secado, Preservación, Descortezado, Partículas). M ontevideo. Hemisferio Sur. 688 pp.

VILLANUEVA， G. 1977. Inventario Forestal de los Bosques del CIEFOR-Pto. Almendras. Iquitos. UNAP. Programa Académico de Ingeniería Forestal. 47pp.

-G. 1982. Inventario Forestal del Bosque Santa Cruz - Río Nanay. Iquitos. Programa A cadémico de Ingeniería F orestal-Instituto de Investigación Forestal y de Fauna. 42 pp.

1984. Inventario de los Bosques de San Juan del Ojeal - Río Amazonas. Iquitos. UNA P-F acultad de Ingeniería Forestal. 48 pp.

VILLAR, E. 1984. Evaluación de dos M étodos de Muestreo en un Bosque Tropical del Distrito de Nauta - Loreto. Tesis Ing. For. Iquitos. Perú. IJ 7NAP. Facultad de Ingeniería Forestal. 61 pp. 